\title{
INFRARED SPECTROSCOPY OF SYMBIOTIC STARS. VIII. ORBITS FOR THREE S-TYPE SYSTEMS: AE ARAE, Y CORONAE AUSTRALIS, AND SS 73-147
}

\author{
Francis C. Fekel ${ }^{1,4}$, Kenneth H. Hinkle ${ }^{2}$, Richard R. Joyce ${ }^{2}$, And Peter R. Wood ${ }^{3}$ \\ ${ }^{1}$ Center of Excellence in Information Systems, Tennessee State University, 3500 John A. Merritt Boulevard, Box 9501, \\ Nashville, TN 37209, USA; fekel@evans.tsuniv.edu \\ 2 National Optical Astronomy Observatory, P.O. Box 26732, Tucson, AZ 85726, USA; hinkle@noao.edu, joyce@noao.edu \\ ${ }^{3}$ Research School of Astronomy and Astrophysics, Mount Stromlo Observatory, Australian National University, \\ Cotter Road, Weston Creek, ACT 2611, Australia; wood@mso.anu.edu.au \\ Received 2009 November 30; accepted 2010 January 14; published 2010 February 19
}

\begin{abstract}
With new infrared radial velocities we have computed orbits of the $M$ giants in three southern S-type symbiotic systems. AE Ara and SS 73-147 have circular orbits with periods of 803 and 820 days, respectively. The eccentric orbit of Y CrA has a period that is about twice as long, 1619 days. Except for $\mathrm{CH}$ Cyg it is currently the S-type symbiotic system with the longest period for which a spectroscopic orbit has been determined. The Paschen $\delta$ emission line velocities of AE Ara are nearly in antiphase with the $\mathrm{M}$ giant absorption feature velocities and result in a mass ratio of 2.7. Emission lines in the $1.005 \mu \mathrm{m}$ region for the other two symbiotic systems are not good proxies for the hot components in those systems. There is no evidence that these three symbiotics are eclipsing. With spectral classes of M5.5 or M6, the three giants presumably also have velocity variations that result from pulsations, but we have been unable to identify specific pulsation periods in the absorption line velocity residuals.
\end{abstract}

Key words: binaries: symbiotic - infrared: stars - stars: individual (AE Ara, Y CrA, SS 73-147) - stars: late-type

\section{INTRODUCTION}

About 25 years ago visual and ultraviolet spectroscopy (Kenyon \& Webbink 1984; Garcia 1986; Mürset et al. 1991) finally demonstrated that symbiotic stars are mass-transfer binaries, consisting of a cool giant and a hot star. The companion of the $\mathrm{K}$ or $\mathrm{M}$ giant is typically a white dwarf and at least in one case (Hinkle et al. 2006) a neutron star, but in a few systems the secondary may be a low mass, main-sequence star. From their characteristics at infrared wavelengths, Webster \& Allen (1975) separated the symbiotics into two subclasses, D for dusty-type and $\mathrm{S}$ for stellar-type systems. The S-type symbiotics have typical orbital periods of 2-3 yr, while those of the D-type systems are at least an order of magnitude longer (Schmid \& Schild 2002). For a more extensive introduction to this group of stars see Fekel et al. (2000b) and Mikołajewska (2003).

Orbital elements of the late-type giant component provide a major starting point for an understanding of symbiotic systems. However, the blue wavelength absorption line spectra are contaminated by veiling and numerous emission features, so absorption lines of the giant component are not generally measurable. To alleviate such difficulties, observations to detect the $\mathrm{K}$ or $\mathrm{M}$ giant component are best made at infrared wavelengths. A second problem is the faintness of the stars. About $50 \%$ of the confirmed symbiotics in the catalog of Belczyński et al. (2000) have $V \geqslant 13.0 \mathrm{mag}$, so in general, moderate aperture telescopes are required to obtain high resolution spectra from which precise radial velocities can be determined. A third impediment is the long orbital periods of the systems, which result in low velocity amplitudes. This difficulty is compounded because many of the cool giants have pulsations that produce significant velocity changes that are evident as larger residuals to orbital fits. These various complications conspire to make

\footnotetext{
4 Visiting Astronomer, Kitt Peak National Observatory, National Optical Astronomy Observatory, operated by the Association of Universities for Research in Astronomy, Inc., under cooperative agreement with the National Science Foundation.
}

well-determined orbital elements difficult to obtain. As a result, of the nearly 200 symbiotic systems listed in the recent catalog of Belczyński et al. (2000), only about $15 \%$ have had spectroscopic orbital elements determined for the cool giant component (Mikołajewska 2003; Fekel et al. 2008). In seven previous papers in this series (Fekel et al. 2000a, 2000b, 2001, 2007, 2008; Hinkle et al. 2006, 2009) we have determined the orbital elements for the late-type component in 21 systems, seven of which are first orbits.

The present work provides the spectroscopic orbits for the late-type giant components in three S-type symbiotics, AE Ara, Y CrA, and SS 73-147. Unless a system has a variable star designation, its names come from various survey lists because of the general faintness of the objects in this class. One of the three systems that we discuss does not have a variable star name, and so we have chosen to use the designation SS 73-147 from the list of Sanduleak \& Stephenson (1973), who were the first to identify the star as a possible symbiotic system, rather than the earlier designation of Hen 2-374 from a survey where the system was identified as a planetary nebula (Henize 1967). The catalog of Belczyński et al. (2000) provides a variety of alternative designations for these objects.

Being located in the southern sky, these symbiotic stars, especially the latter two, have not been as extensively observed and analyzed as most of their northern cousins. It is hoped that the determination of the orbits and the discussion in this paper will lead to more intensive observing of these symbiotics. Some basic information for the three systems is provided in Table 1.

\section{OBSERVATIONS AND REDUCTIONS}

From 2001 March to 2009 June we obtained 22 spectroscopic observations of AE Ara. The first eight were acquired with the $1.88 \mathrm{~m}$ telescope and coudé spectrograph system at the Mount Stromlo Observatory (MSO), Canberra, Australia. The detector was an infrared camera, NICMASS, developed at the University of Massachusetts. We obtained a 2 pixel resolving power of 44,000 at a wavelength of $1.623 \mu \mathrm{m}$. A more 
Table 1

Basic Properties of The Program Stars

\begin{tabular}{|c|c|c|c|c|c|c|}
\hline Name & $\begin{array}{c}V \\
(\mathrm{mag}) \\
\end{array}$ & $\begin{array}{c}K \\
(\mathrm{mag}) \\
\end{array}$ & $\begin{array}{l}H-K \\
(\mathrm{mag}) \\
\end{array}$ & $\begin{array}{c}\text { Primary } \\
\text { Spectral Class }^{\mathrm{b}}\end{array}$ & $\begin{array}{c}\text { Orbital Period } \\
\text { (days) }\end{array}$ & $\begin{array}{c}\dot{M}^{\mathrm{a}} \\
\left(M_{\odot} \mathrm{yr}^{-1}\right) \\
\end{array}$ \\
\hline AE Ara & $12.14^{\mathrm{c}}-13.6^{\mathrm{d}}$ & $6.48^{c}$ & $0.30^{\mathrm{c}}$ & M5.5 & 803 & $\ldots$ \\
\hline Y CrA & $14.35^{\mathrm{c}}-15.5^{\mathrm{d}}$ & $6.48^{\mathrm{e}}$ & $1.29^{\mathrm{e}}$ & M6 & 1619 & $3.4 \times 10^{-7}$ \\
\hline SS 73-147 & $\sim 13.6^{\mathrm{f}}$ & $6.55^{\mathrm{g}}$ & $0.41^{\mathrm{g}}$ & M5.5 & 820 & $\ldots$ \\
\hline
\end{tabular}

Notes. ${ }^{\text {a }}$ Seaquist et al. 1993; ${ }^{\mathrm{b}}$ Mürset \& Schmid 1999; ${ }^{\mathrm{c}}$ Munari et al. $1992 ;{ }^{\mathrm{d}}$ Gutierrez-Moreno et al. 1999; ${ }^{\mathrm{e}}$ Phillips 2007; ${ }^{\mathrm{f}}$ Pojmanski 2002; ${ }^{\mathrm{g}}$ Kenyon \& Gallagher 1983.

complete description of the experimental setup may be found in Joyce et al. (1998), as well as in Fekel et al. (2000b). The detector and electronics were previously used for our survey of northern symbiotics, carried out with the coudé feed telescope at Kitt Peak National Observatory (KPNO).

The major Canberra area bush fires of 2003 January caused the destruction of both the $1.88 \mathrm{~m}$ telescope at MSO and our infrared NICMASS camera, resulting in the premature conclusion of our observing program at that observatory. To continue our project, on three dates between 2003 February and 2004 April we observed AE Ara with the Phoenix cryogenic echelle spectrograph, mounted on the $8 \mathrm{~m}$ Gemini South telescope at Cerro Pachon, Chile. A complete description of the spectrograph can be found in Hinkle et al. (1998). The first Gemini South observation was centered at $1.563 \mu \mathrm{m}$ and had a resolving power of 50,000. Two other spectra were obtained at $2.224 \mu \mathrm{m}$, a region containing several atomic lines, and have a resolving power of $\sim 70,000$. That wavelength and resolution were selected to allow detailed analysis of line profiles. These data were taken for a separate program on symbiotic abundances as well as the current radial velocity work.

Our more recent observations were acquired at KPNO, which at latitude $+32^{\circ}$ is situated well above the equator in the northern hemisphere. With a declination of $-47^{\circ} \mathrm{AE}$ Ara rises just $11^{\circ}$ above the horizon, making observations very challenging. Its short window of observability from that observatory requires nearly ideal observing conditions to obtain useful spectra. Fortunately, we have been successful more often than not. Nine observations were obtained from 2004 May to 2009 June at KPNO with the 0.9 m coudé feed telescope, coudé spectrograph, and a CCD, designated LB1A. This $1980 \times 800$ pixels CCD was manufactured by Lawrence Berkeley National Laboratory and is $300 \mu \mathrm{m}$ thick. Although this thickness results in increased pixel contamination by cosmic ray and background radiation events, the chip was used because of its high quantum efficiency at far red wavelengths. Our spectrograms, centered near $1.005 \mu \mathrm{m}$, have a wavelength range of $420 \AA$ and a resolving power of $\sim 21,500$.

Finally, in 2009, we obtained observing time for our project on the $1.5 \mathrm{~m}$ telescope at the Cerro Tololo Inter-American Observatory. The telescope is operated by the Small and Moderate Aperature Research Telescope System (SMARTS) consortium of universities and other organizations. From 2009 May through July we obtained two spectra of AE Ara with that telescope, a fiber fed echelle spectrograph, and a 2K SITe CCD. The spectra have a resolving power of $\sim 25,000$ at $5500 \AA$.

The observing history of Y CrA is similar to that of AE Ara. Over an eight-year period we collected 20 spectroscopic observations of Y CrA. From 2001 May through 2002 August, we acquired seven spectrograms at MSO with the same telescope, spectrograph, and infrared camera that were used for AE Ara.
Shortly after the loss of our instrument and the MSO facility, we obtained a single observation with the $8 \mathrm{~m}$ Gemini South telescope and the Phoenix spectrograph. We continued our observations at KPNO with nine more spectrograms being acquired from 2004 May to 2009 June. For those observations we used the coudé feed telescope, coudé spectrograph, and LB1A CCD detector. Also in 2009, we obtained two observations with the $1.5 \mathrm{~m}$ SMARTS telescope and echelle spectrograph plus a single spectrogram with the Gemini South telescope and Phoenix spectrograph.

The 16 spectra that we collected of our third symbiotic, SS 73-147, were obtained at the first three observatories, mentioned above, and with the same instruments and detectors. The first six observations were acquired from 2001 May through 2002 August at MSO. A single Gemini South spectrum in 2004 was followed by nine observations at KPNO that were obtained between 2005 June and 2009 June.

We have used standard observing and reduction techniques (Joyce 1992). However, wavelength calibration at the infrared wavelengths of 1.563 and $2.224 \mu \mathrm{m}$ was problematic, because the spectral coverage was far too small to include a sufficient number of ThAr emission lines for a dispersion solution. Thus, at $1.563 \mu \mathrm{m}$ we instead utilized the absorption lines of a bright late-type giant star, usually $\delta$ Oph. Approximately 20 lines of $\mathrm{CN}, \mathrm{Fe}$, and $\mathrm{OH}$ were used to calculate the dispersion solution in this wavelength region. Each solution was offset to an absolute standard provided by one or more telluric $\mathrm{OH}$ emission lines. At $2.224 \mu \mathrm{m}$ the wavelength calibration was done by measuring telluric absorption lines in the spectrum of $\beta$ Lup, a bright earlytype star. The typical rms of the solutions for both regions was about $0.02 \AA$.

For the spectrograms acquired with the LB1A CCD at $1.005 \mu \mathrm{m}$, and the optical wavelength echelle spectrograms from the $1.5 \mathrm{~m}$ SMARTS telescope, we were able to use ThAr spectra for wavelength calibration. Telluric lines are present in the 1.005 and $2.224 \mu \mathrm{m}$ wavelength regions. These lines were removed from our observations by ratioing the spectra to a hot star spectrum observed on the same night.

The echelle spectrograms from the $1.5 \mathrm{~m}$ SMARTS telescope cover the wavelength range 4020-7300 A. Given the difficulty of measuring the $\mathrm{M}$ giant absorption line spectrum at blue wavelengths, we have chosen to measure velocities in one of the two reddest orders of the echelle spectrum, which is centered at $7130 \AA$ A Telluric lines are generally weak in this region, and so we did not attempt to remove them from the measured spectra.

Representative spectra of the program stars at wavelengths of $1.005,1.563$, and $2.224 \mu \mathrm{m}$ are shown in Figures 1-3, respectively. No spectrum of SS 73-147 was obtained at $1.563 \mu \mathrm{m}$, and so Figure 2 shows the spectra of only Y CrA and AE Ara. Spectra of our velocity standard $\delta$ Oph, which has a spectral type of M0.5 III (Keenan \& McNeil 1989), have been 


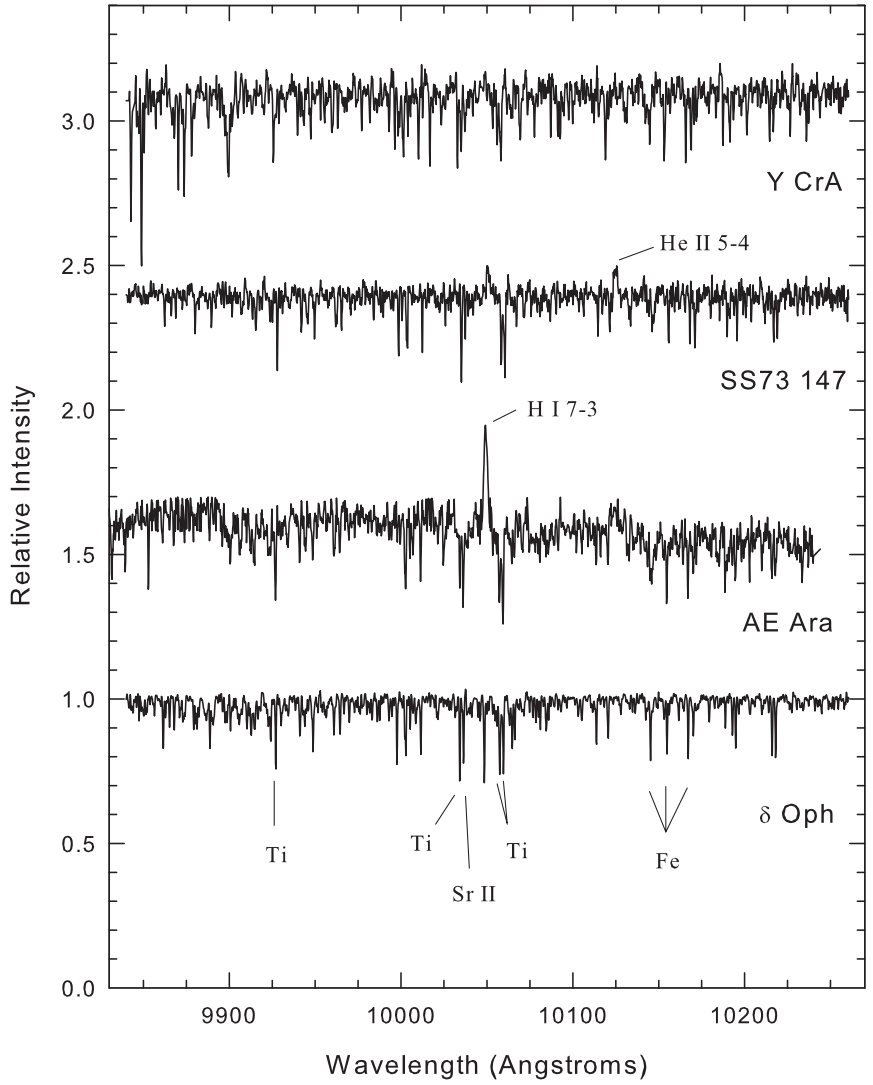

Figure 1. Spectra of the three program stars and velocity standard $\delta$ Oph at $1.005 \mu \mathrm{m}$ observed with the KPNO coudé feed telescope, spectrograph, and LB1A CCD. The entire observed spectral region is shown. The telluric spectrum has been ratioed out by referencing a hot star spectrum acquired on the same night. The relative intensity scales for AE Ara, SS 73-147, and Y CrA have been offset by $0.6,1.4$, and 2.1 , respectively. A few of the stronger representative absorption and emission lines are identified.

included for comparison in each of the three figures. In all three regions, the vast majority of the lines arise in the photosphere of the red giant. The $1.005 \mu \mathrm{m}$ region contains several neutral atomic lines, especially from $\mathrm{Ti}$ and $\mathrm{Fe}$. There are also many weak to moderate strength lines from bands of the $\Delta v=-1 \mathrm{CN}$ red system. The $1.563 \mu \mathrm{m}$ region is dominated by $\mathrm{OH}$ first overtone lines and a selection of neutral atomic lines and also has weak $\mathrm{CN}$ red system $\Delta v=-1$ lines and $\mathrm{CO}$ vibration-rotation second overtone lines. The $2.224 \mu \mathrm{m}$ region contains moderately strong Ti I lines as well as a few other neutral atomic lines, especially from $\mathrm{Fe}$ I and $\mathrm{Sc}$. The $\mathrm{CN}$ red-system $\Delta v=-2$ transition provides a background of weak lines.

Unlike the two spectral regions farther to the red, several emission features are obvious in our spectra of the $1.005 \mu \mathrm{m}$ region (Figure 1). The two that are generally strongest in the spectra of our three stars are the Paschen $\delta$ line at $1.004938 \mu \mathrm{m}$ and the He II line at $1.012361 \mu \mathrm{m}$. A third emission feature that appears in some spectra is the Fe II line at $0.999756 \mu \mathrm{m}$, which has been found previously in the infrared spectra of $\eta \mathrm{Car}$ (Johansson 1977) and Be stars (Andrillat et al. 1990).

Absorption line radial velocities of the program stars were measured with the IRAF cross-correlation program FXCOR (Fitzpatrick 1993). The velocities were determined relative to the M giant International Astronomical Union velocity standard $\delta$ Oph, which was observed multiple times during the course of each night. Its radial velocity of $-19.1 \mathrm{~km} \mathrm{~s}^{-1}$ was adopted from the work of Scarfe et al. (1990).

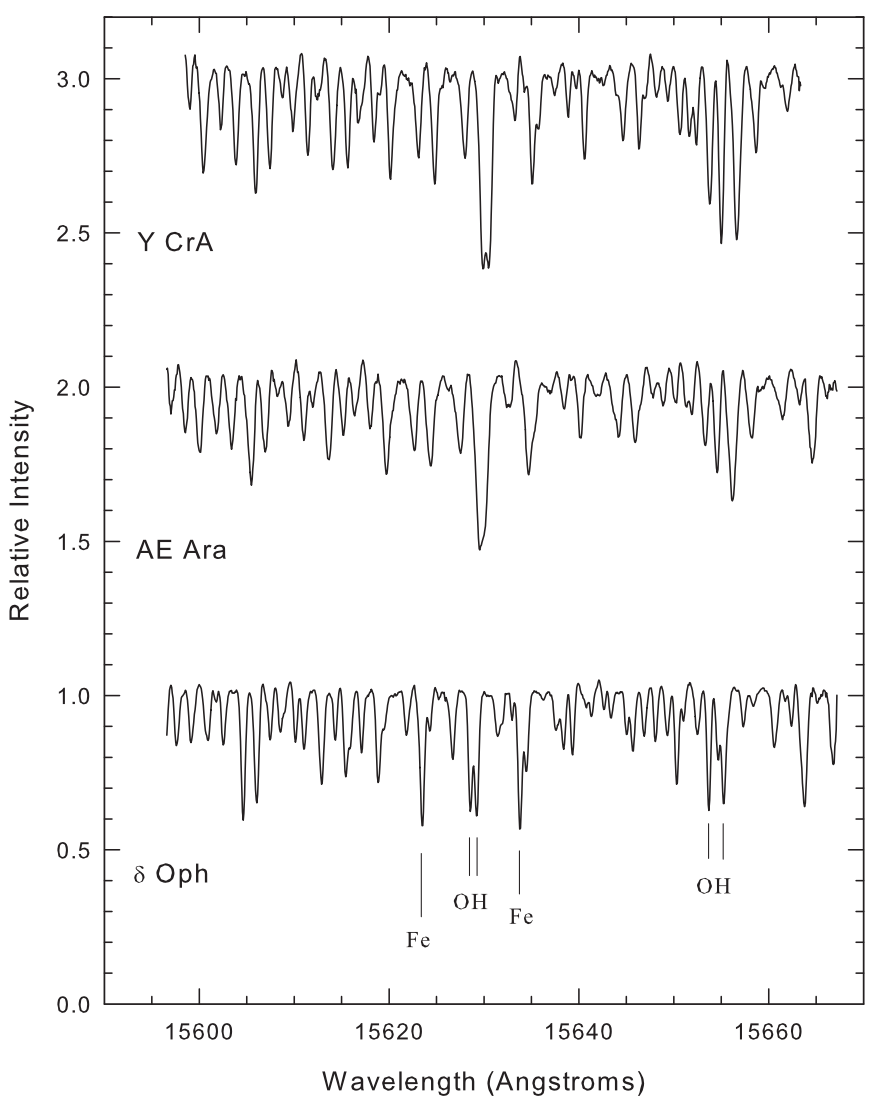

Figure 2. Spectra of AE Ara, Y CrA, and $\delta$ Oph at $1.563 \mu \mathrm{m}$ observed with the Phoenix spectrograph on the Gemini South telescope. The relative intensity scales for AE Ara and Y CrA have been offset by 1.0 and 2.0, respectively. No correction for telluric absorption lines is needed in this spectral region.

Radial velocities of the Paschen $\delta$ and He II emission lines were measured with the IRAF task RVIDLINES. A Gaussian function was fitted to each emission line, and the wavelength shift of the spectral feature relative to its rest wavelength was converted into a radial velocity, which was then corrected for Earth's motion.

\section{ORBITAL ANALYSIS}

Preliminary orbital periods for the red giants were determined with a program named PGRAM (PeriodoGRAM). An extensive range of periods was searched by computing the phases of the observed velocities for each trial period and comparing those phased velocities to a sine curve fit. The sum of the squared velocity residuals from the sine curve fit was computed, and the period having the smallest value of that sum was adopted as the best preliminary period. With this period identified, spectroscopic orbital elements of the systems were determined with various computer programs (Fekel et al. 2008) for eccentric and circular orbits.

\section{PULSATION PERIOD SEARCH}

As summarized by Percy et al. (2001), all $\mathrm{M}$ giants have light variability from pulsation at some level and the amplitude of such variability generally increases with later spectral type and higher luminosity. Variables with amplitudes less than $2.5 \mathrm{mag}$ are classified as semi-regular. Lebzelter \& Hinkle (2002) noted that the periods of these semi-regular variables 


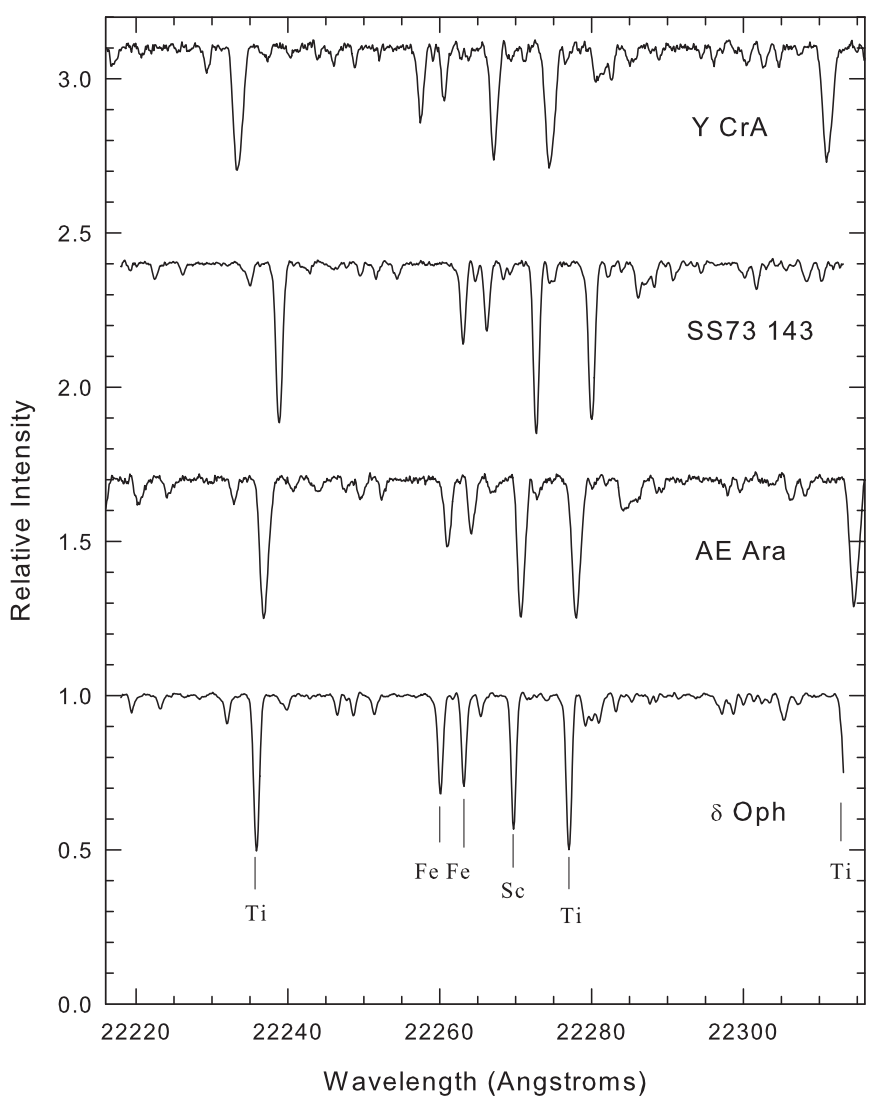

Figure 3. Spectra of the three program stars and $\delta$ Oph at $2.227 \mu \mathrm{m}$ observed with the Phoenix spectrograph on the Gemini South telescope. The relative intensity scales for AE Ara, SS 73-147, and Y CrA have been offset by $0.7,1.4$, and 2.1, respectively. The telluric spectrum has been ratioed out by referencing a hot star spectrum acquired on the same night.

typically range from 30 to 200 days. Velocity variations in semiregular variables accompany the light variability and generally display amplitudes of up to a few $\mathrm{km} \mathrm{s}^{-1}$ (e.g., Lebzelter \& Hinkle 2002). Thus, given the M5-M6 spectral classes of the giants in our three symbiotic binaries, a portion of the radial velocity variations results from pulsation. Comparing the results for the three systems in this paper with our symbiotic orbits with the smallest velocity residuals (Fekel et al. 2000b, 2001), we estimate that at least half of the rms error found for the radial velocity orbital solutions of AE Ara, Y CrA, and SS 73-147 results from pulsation.

Thus, after determining orbital elements for each red giant, we also performed period searches of the residuals of our adopted absorption-line radial velocity solutions with PGRAM. However, we caution that the number and distribution of our observations are not well suited to identifying pulsation periods. This is because the frequency of our velocity observations, two or three per year for several years, produces a total of just 16-22 velocities that are poorly spaced to determine periods of weeks or several months. In addition, the amplitudes of our best periods are very low, only $2-4 \mathrm{~km} \mathrm{~s}^{-1}$, so the best periods are heavily dependent on the placement of the few velocities with the largest residuals. In the sections for the individual stars we discuss our specific results, but we generally conclude that although our three giants appear to have velocity variability due to pulsation, it is impossible to determine the true periods from our limited number of velocities and thus, remove the pulsational variations from our data.
5. AE Ara $=$ Hen 3-1451 = SS 73-95 = Wray $15-1754$

\subsection{Short History}

Cannon (1933) announced the discovery of five new variable stars with strong hydrogen emission lines. The one designated Harvard variable (HV) 5491 and later given the variable star name AE Ara was described as having a peculiar spectrum reminiscent of a nova. Analysis of Harvard College Observatory photographic plates established that the light variations are irregular in character. Those changes included sudden brightness increases of over $1.5 \mathrm{mag}$ during the fall of 1913 and again during the spring of 1931.

Further analysis of its light variations languished until Mikołajewska et al. (2003) published an abbreviated report of observations from the 1980s and 1990s. They analyzed visual photometry from the Variable Star Section Circulars of the Royal Astronomical Society of New Zealand. The visual light curve showed that the star was quiescent for much of the period covered by the observations, and Mikołajewska et al. (2003) were able to identify a periodic variation of about 800 days that had an amplitude of $0.4 \mathrm{mag}$.

Skopal et al. (2007) added more recent visual measurements to the time series presented by Mikołajewska et al. (2003). Skopal et al. (2007) stated that the best defined minimum in their newer data agreed with the ephemeris of Mikołajewska et al. (2003). They also noted that at the beginning of $2006 \mathrm{AE}$ Ara entered a new active phase, but the increased light output had begun to decline by the end of the year.

After its discovery as a variable star, it took decades before the true nature of $\mathrm{AE}$ Ara was revealed, and there were several false steps along the way. Merrill \& Burwell (1943) included the star in a list of B and A stars with hydrogen emission lines. Peimbert \& Costero (1961) examined both their Tonantzintla direct plates and their objective prism plates of the object. They characterized its image as stellar, and it received the designation of PC 18. Its objective prism spectrum showed no continuum, but Peimbert \& Costero (1961) did detect emission lines of $\mathrm{H} \beta$ and $\mathrm{H} \gamma$ as well as two nebular lines, and so they identified $\mathrm{AE}$ Ara as a planetary nebula.

In the late 1940s and early 1950s, Henize (1967) went to South Africa and obtained objective-prism spectra of the sky south of declination $-25^{\circ}$ to identify $\mathrm{H} \alpha$ emission-line objects. From the long-exposure plates of that survey Wray (1966) confirmed the $\mathrm{H} \alpha$ emission, and the star was identified as Wray 15-1754. Henize (1976) published a list of over 1900 southern, $\mathrm{H} \alpha$ emission-line stars, found from his objective-prism spectra. Designated as Hen 3-1451, its spectrum was simply called peculiar.

Sanduleak \& Stephenson (1973) carried out an extensive lowdispersion objective-prism survey of the southern Milky Way, looking for various types of strong emission-line objects. For AE Ara, which was number 95 in their 1973 list, they detected only hydrogen emission, and because those lines were extremely strong, they classified it as an extreme Be-like object, which they designated as Be!.

Allen \& Glass (1974) obtained infrared photometry of a variety of southern emission-line stars. They concluded from their infrared colors of AE Ara that it is not a Be star but rather is a symbiotic star. Following up on the work of Sanduleak \& Stephenson (1973), Allen (1978) acquired a low-dispersion slit spectrum of AE Ara. He characterized the star as probably having weak emission lines of high excitation and a strong unidentified band at $6830 \AA$ A. Later, Schmid (1989) identified 
Table 2

Absorption Line Radial Velocities of AE ARA

\begin{tabular}{lcccc}
\hline \hline $\begin{array}{c}\text { HJD } \\
(2400000+)\end{array}$ & Phase & $\begin{array}{c}\text { Velocity } \\
\left(\mathrm{km} \mathrm{s}^{-1}\right)\end{array}$ & $\begin{array}{c}O-C \\
\left(\mathrm{~km} \mathrm{~s}^{-1}\right)\end{array}$ & Observatory $^{\mathrm{a}}$ \\
\hline 51993.263 & 0.938 & -7.9 & -3.3 & MSO \\
52046.181 & 0.004 & -4.3 & -0.1 & MSO \\
52094.211 & 0.064 & -4.9 & -0.2 & MSO \\
52131.098 & 0.110 & -5.1 & 0.6 & MSO \\
52356.238 & 0.390 & -16.0 & -0.2 & MSO \\
52398.320 & 0.443 & -16.8 & 0.0 & MSO \\
52445.172 & 0.501 & -18.0 & -0.7 & MSO \\
52503.983 & 0.574 & -18.0 & -1.4 & MSO \\
52687.884 & 0.803 & -7.9 & 0.7 & Gemini S \\
52749.868 & 0.880 & -2.7 & 3.2 & Gemini S \\
53098.849 & 0.314 & -14.8 & -1.5 & Gemini S \\
53131.946 & 0.356 & -15.0 & -0.3 & KPNO \\
53538.820 & 0.862 & -6.9 & -0.4 & KPNO \\
53899.827 & 0.311 & -10.1 & 3.1 & KPNO \\
54231.940 & 0.725 & -12.3 & -0.6 & KPNO \\
54270.813 & 0.773 & -9.8 & 0.0 & KPNO \\
54592.932 & 0.174 & -8.9 & -1.2 & KPNO \\
54634.831 & 0.226 & -8.7 & 1.0 & KPNO \\
54959.944 & 0.631 & -14.7 & 0.5 & KPNO \\
54976.873 & 0.652 & $-13.4^{\mathrm{b}}$ & 1.1 & CTIO \\
54998.828 & 0.679 & -12.5 & 1.0 & KPNO \\
55037.706 & 0.728 & $-9.9 \mathrm{~b}$ & 1.7 & CTIO \\
\hline & & & &
\end{tabular}

Notes.

${ }^{a}$ MSO, Mount Stromlo Observatory; Gemini S, Gemini South Observatory; KPNO, Kitt Peak National Observatory; CTIO, Cerro Tololo Inter-American Observatory.

b Velocity given zero weight.

that emission band as the result of Raman scattering of the $\mathrm{O}$ VI resonance lines by neutral hydrogen.

Ultimate confirmation of the $M$ giant spectrum in AE Ara came from Allen (1980), who estimated a spectral class of M2 for AE Ara from a spectrum at $2 \mu \mathrm{m}$. Medina Tanco \& Steiner (1995) classified the star as M5 from a visual comparison of its red wavelength region with standard star spectra. Mikołajewska et al. (1997) found a spectral class of M3, but they noted that their spectral class must be considered an upper limit, and a later subclass was likely. Gutierrez-Moreno et al. (1999) found a spectral class of M4.4, using near-infrared molecular band indices, while Mürset \& Schmid (1999) determined a slightly later class of M5.5, from an analysis of the strength of several near-infrared wavelength $\mathrm{TiO}$ bandheads. In addition to their spectral class of the red giant, Mikołajewska et al. (1997) provided a limited analysis of AE Ara, estimating the hot component temperature and luminosity plus the system distance.

AE Ara was included in the symbiotic star catalog of Allen (1984), where a low-dispersion spectrum was shown, as well as the more recent catalog of Belczyński et al. (2000). The latter listed the spectroscopic orbital elements of 20 symbiotics, but AE Ara was not one of those stars. However, Mikołajewska et al. (2003) summarized results of a decade long spectroscopic campaign on AE Ara. They found its M giant component to have an orbital period of 812 days. They also determined velocities of a broad helium emission line and then estimated the mass ratio of the $\mathrm{M}$ giant and hot companion.

\subsection{Spectroscopic Orbit}

From 2001 March to 2009 July we obtained 22 absorption line radial velocities of AE Ara (Table 2). However, the two

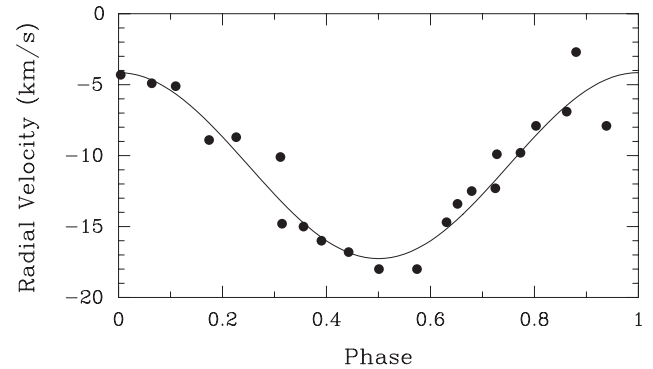

Figure 4. $M$ giant radial velocities of AE Ara (filled circles) compared with its computed circular orbit (solid line). Zero phase is a time of maximum velocity.

Table 3

Orbital Elements and Related Parameters of AE ARA

\begin{tabular}{|c|c|c|}
\hline Parameter & $\begin{array}{l}\text { This } \\
\text { Paper }\end{array}$ & $\begin{array}{l}\text { Milolajewska et al. } \\
\qquad(2003)\end{array}$ \\
\hline$P$ (days) & $803.4 \pm 8.8$ & $812 \pm 2$ \\
\hline$T_{0}(\mathrm{HJD})$ & $2453649.6 \pm 9.7$ & $2450217^{\mathrm{a}} \pm 3$ \\
\hline$\gamma\left(\mathrm{km} \mathrm{s}^{-1}\right)$ & $-10.70 \pm 0.36$ & $-15.7 \pm 0.3$ \\
\hline$K\left(\mathrm{~km} \mathrm{~s}^{-1}\right)$ & $6.55 \pm 0.55$ & $5.4 \pm 0.3$ \\
\hline$e$ & 0.0 (adopted) & 0.0 (adopted) \\
\hline$a \sin i\left(10^{6} \mathrm{~km}\right)$ & $72.41 \pm 6.14$ & $\ldots$ \\
\hline$f(m)\left(M_{\odot}\right)$ & $0.0235 \pm 0.0059$ & $\ldots$ \\
\hline $\begin{array}{l}\text { Standard error of an observation } \\
\text { of unit weight }\left(\mathrm{km} \mathrm{s}^{-1}\right)\end{array}$ & 1.6 & $\ldots$ \\
\hline
\end{tabular}

Note. ${ }^{\text {a }}$ M III superior conjunction.

SMARTS spectra had very low signal-to-noise ratios, and so those velocities were given weights of zero in our analyses. The remaining 20 velocities produced an initial period of 803 days, in reasonable agreement with the value of 812 days found by Mikołajewska et al. (2003) for their circular orbit solution. Thus, our observations cover about 3.7 orbital cycles. Adopting the period of 803 days, we obtained preliminary orbital elements, which were then refined. That second solution produced an orbit with a period of 803 days and an eccentricity of $0.098 \pm 0.079$. Because of this low orbital eccentricity and its large uncertainty, we also determined a circular-orbit solution, which has a period of 803 days. The tests of Lucy \& Sweeney (1971) indicated that the circular orbit solution is to be preferred, so it is listed in Table 3. A time of periastron passage, $T$, is undefined for a circular orbit. Thus, as recommended by Batten et al. (1989), we have instead listed $T_{0}$, a time of maximum velocity, in the table of orbital elements. The standard error of an individual velocity is $1.6 \mathrm{~km} \mathrm{~s}^{-1}$. This high value results from the large residuals (Table 2) and argues that there is also a pulsational component in the velocities. Our 22 radial velocities are compared to the computed circular-orbit velocity curve in Figure 4.

\subsection{Emission Line Spectrum and Orbit}

Symbiotics in general display many strong emission lines from an ionized nebula (Mikołajewska 2003), and several nebular regions are usually postulated including one surrounding the hot component (e.g., Dobrzycka et al. 1993). Thus, it is possible that some emission lines may at least partially reflect the motion of the secondary.

Having determined our orbit for the $\mathrm{M}$ giant, we examined the velocities of the Paschen $\delta$ and He II emission lines in the $1 \mu \mathrm{m}$ region. The Paschen $\delta$ lines are reasonably strong and relatively symmetric. Most of the He II lines are weak but also reasonably symmetric. We list the velocities of the two emission lines in Table 4. 
Table 4

Emission Line Radial Velocities of AE ARA

\begin{tabular}{ccrcrc}
\hline \hline $\begin{array}{c}\text { HJD } \\
(2400000+)\end{array}$ & Phase & $\begin{array}{c}\text { H I } \\
\text { Velocity } \\
\left(\mathrm{km} \mathrm{s}^{-1}\right)\end{array}$ & $\begin{array}{c}\text { He II } \\
\text { Velocity } \\
\left(\mathrm{km} \mathrm{s}^{-1}\right)\end{array}$ & $\begin{array}{c}\text { HI } \\
O-C \\
\left(\mathrm{~km} \mathrm{~s}^{-1}\right)\end{array}$ & $\begin{array}{c}\text { He II } \\
O-C \\
\left(\mathrm{~km} \mathrm{~s}^{-1}\right)\end{array}$ \\
\hline 53131.946 & 0.356 & 2.2 & -15.2 & 2.1 & -15.3 \\
53538.820 & 0.862 & -28.5 & -21.9 & -6.4 & 0.2 \\
53899.827 & 0.311 & 2.0 & -24.3 & 6.1 & -20.2 \\
54231.940 & 0.725 & -12.3 & 14.2 & -4.4 & 22.1 \\
54270.813 & 0.773 & -14.5 & 19.7 & -1.2 & 33.0 \\
54592.932 & $0.174^{\mathrm{a}}$ & -12.6 & $\ldots$ & 6.2 & $\ldots$ \\
54634.831 & $0.226^{\mathrm{a}}$ & -8.8 & $\ldots$ & 4.5 & $\ldots$ \\
54959.944 & 0.631 & -4.2 & 7.1 & -5.4 & 5.9 \\
54998.828 & 0.679 & -3.1 & 21.0 & 0.1 & 24.2 \\
\hline
\end{tabular}

Note. ${ }^{\text {a }}$ The He II line is not in emission at this phase.

In Figure 5, the computed $\mathrm{M}$ giant orbit is compared with the two sets of emission line velocities. While our nine emission line spectra span 2.3 orbital cycles, they do not cover the phases at velocity maximum and minimum. Figure 5 also shows that the velocities of the $\mathrm{HI}$ and $\mathrm{He}$ II emission lines are quite different at each phase. Although Mikołajewska et al. (1989) stated that in symbiotics the He II emission lines should follow the hot component, it is clear that in the case of AE Ara the He II emission line at $1.0123 \mu \mathrm{m}$ is not a good proxy for the hot component. The velocities for that line are not in antiphase with the $\mathrm{M}$ giant orbit, and in addition, $\mathrm{He}$ II emission is absent from spectra at phases 0.17 and 0.22 , which correspond to the hot component approaching its superior conjunction. Instead, it is the Paschen $\delta$ emission line velocities that appear to be nearly $180^{\circ}$ out of phase with the M giant orbit.

Thus, we obtained a circular orbit solution for Paschen $\delta$ in which we adopted our orbital period of the $\mathrm{M}$ giant and solved for the other orbital elements. That emission line orbit is nearly $180^{\circ}$ out of phase with the $\mathrm{M}$ giant orbit, differing from that value by less than $15^{\circ}$ or 0.04 phase units. The center-of-mass velocity of the emission line orbit is $-10.1 \pm 1.0 \mathrm{~km} \mathrm{~s}^{-1}$, which is in excellent agreement with the systemic velocity of the $\mathrm{M}$ giant orbit, differing by less than $1.0 \mathrm{~km} \mathrm{~s}^{-1}$. The resulting mass ratio for the red giant and putative white dwarf, $M_{\mathrm{rg}} / M_{\mathrm{wd}}$, is $2.7 \pm 0.4$, a value that is similar to that suggested for most symbiotics (Mikołajewska 2003).

\subsection{Orbital Element Comparison}

Mikołajewska et al. (2003) have previously determined a circular orbit for AE Ara and provided a very brief discussion of their results. They used the velocities of the $\mathrm{M}$ giant absorption lines to determine the orbital elements of the primary. For the secondary, they argued that the velocities from the broad emission line wings of the He II emission line at $4686 \AA$ produced an acceptable orbit. In Table 3, we compare our set of M giant orbital elements with those found by Mikołajewska et al. (2003). Although the two orbital periods are in reasonable agreement, other elements are not particularly consistent. The center-of-mass velocities of the two solutions differ by $5 \mathrm{~km} \mathrm{~s}^{-1}$. The semi-amplitudes are also significantly different, our value being $22 \%$ larger, and the semi-amplitudes do not overlap even after taking their uncertainties into account.

Having argued that their $\mathrm{He}$ II emission line velocities produce an acceptable orbit for the secondary, Mikołajewska et al. (2003) then went on to use the semi-amplitude from that orbit to determine a mass ratio of 4.4 for the system. However, their

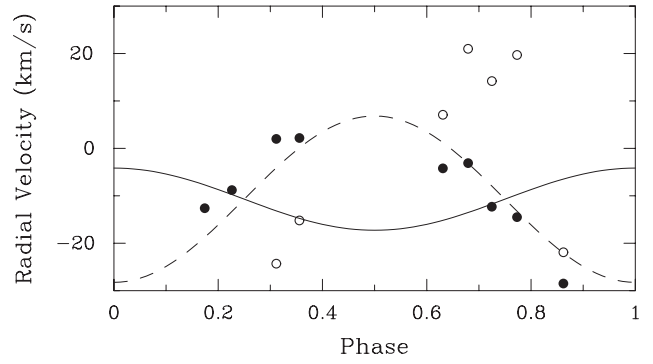

Figure 5. Emission line radial velocities of $\mathrm{AE}$ Ara (filled circles $=\mathrm{HI}$, open circles $=\mathrm{He}_{\mathrm{II}}$ ) compared to the orbit determined for the $\mathrm{M}$ giant (solid line) and the forced fit curve for the $\mathrm{H}$ I emission lines (dashed line), which requires their orbit to be $180^{\circ}$ out of phase with the $\mathrm{M}$ giant orbit. Zero phase is a time of maximum velocity of the $\mathrm{M}$ giant.

emission line center-of-mass velocity differs from that of the $\mathrm{M}$ giant by more than $22 \mathrm{~km} \mathrm{~s}^{-1}$. In addition, although the quiescent state emission velocities are roughly in anti-phase with the $\mathrm{M}$ giant absorption lines, the eight emission line velocities have a huge velocity scatter. Thus, the use of their He II emission-line semi-amplitude for the mass ratio appears to be questionable at best.

\subsection{Pulsation Period Search}

As noted in Section 5.2, from the analysis of the red giant orbit, the standard error of a radial velocity of unit weight is $1.6 \mathrm{~km} \mathrm{~s}^{-1}$, which is 2-3 times larger than the value found for several other symbiotic orbits in our series (e.g., Fekel et al. 2001). Thus, the velocity residuals of the adopted circular orbit were searched for periods between 10 and 1000 days. For the best potential pulsation period of 45.71 days, 18 of the 20 velocities cover only half of the phase curve, and its phase plot is not particularly convincing.

\subsection{Discussion}

$\mathrm{AE}$ Ara has been part of various photometric and spectroscopic surveys. Allen \& Glass (1974) provided infrared J H KL photometric magnitudes, while Munari et al. (1992) determined both $U B V R_{c} I_{c}$ and infrared $J H K L$ magnitudes. As noted in Section 4.1, several groups have determined the spectral class of the late-type component, which has ranged from M2 to M5.5. Mürset \& Schmid (1999) presented the most comprehensive list of spectral classes, deriving results for about 100 systems and including classifications of an additional 70 or so symbiotics from the literature. The low-resolution spectra of Mürset \& Schmid (1999) covered the near-infrared region, where the spectrum of the late-type giant is less affected by veiling than in the visual region. They stated an uncertainty of one spectral subclass for their results, and so we have adopted their classification of M5.5 (Table 1). From a low-resolution spectrogram, Mikołajewska et al. (1997) provided a limited analysis of AE Ara. They estimated a system distance of $2.3 \mathrm{kpc}$ and a hot component temperature between 54,000 and $100,000 \mathrm{~K}$ plus a luminosity of $1000 L_{\odot}$.

Based on the theoretical work of Zahn (1977), Schmutz et al. (1994) and Mürset et al. (2000) have argued that in most S-type symbiotics the giant star is synchronously rotating. They then derived the radius of the giant in various symbiotic binaries.

If, as suggested by theory, the $M$ giant is synchronously rotating, its projected rotational velocity can be used to estimate its minimum radius. To determine $v \sin i$ of the $\mathrm{M}$ giant, we 
measured the full-width at half-maximum of a few atomic features observed on the Phoenix $2.224 \mu \mathrm{m}$ spectra. We also measured the same lines in several late-type giants with known $v \sin i$ values. With the latter set of stars, we produced an empirical broadening calibration similar to that of Fekel (1997). Using the calibration and an adopted macroturbulence of $3 \mathrm{~km} \mathrm{~s}^{-1}$, we determined $v \sin i=8 \pm 1 \mathrm{~km} \mathrm{~s}^{-1}$ from a single observation.

The synchronous period and the $v \sin i$ value result in a minimum radius (i.e., $\sin 90^{\circ}=1$ ) of $127 \pm 16 R_{\odot}$. If, as is generally assumed, the orbital inclinations of binaries are randomly oriented in space, then half of the inclinations are greater than $60^{\circ}$ and half are less than this value (Russell et al. 1938). In addition, if, as expected, the orbital and rotational axes of the binaries are parallel, then the same distribution of inclinations holds for the rotational inclinations. Reducing the rotational inclination from $90^{\circ}$, an angle making the rotational axis perpendicular to the viewing direction, to $60^{\circ}$ increases the radius to $147 R_{\odot}$.

The computed minimum radius can be compared to the Roche lobe radius of the giant. The latter depends on the separation of the stars and their mass ratio. Mikołajewska (2003) summarized estimates of the mass ratios and masses of symbiotic systems. She found that the vast majority of the mass ratios for S-type symbiotics range from 2 to 4 , and average masses for the cool giant and hot component were 1.7 and $0.53 M_{\odot}$, respectively. Guided by those results and our estimated mass ratio of 2.7 , we have adopted masses of 1.6 and $0.6 M_{\odot}$ for the $\mathrm{M}$ giant and its companion, respectively. We then used Kepler's third law to determine the semimajor axis of the orbit. With Equation (2) of Eggleton (1983), we computed a Roche-lobe radius of $221 R_{\odot}$ for the $\mathrm{M}$ giant, which is almost $50 \%$ larger than our minimum radius. A rotational inclination of about $35^{\circ}$ is needed for the $\mathrm{M}$ giant radius to fill its Roche lobe.

From our adopted spectral class of M5.5 (Mürset \& Schmid 1999), we assume an effective temperature of 3425K (Dyck et al. 1996). That, combined with our minimum radius, results in a luminosity of $1989 L_{\odot}$, leading to $M_{\mathrm{bol}}=-3.5 \mathrm{mag}$, while if the red giant fills its Roche lobe radius, its total brightness would be increased by $1.2 \mathrm{mag}$.

To estimate the minimum distance to AE Ara, we adopted its $K$ mag and $J-K$ color from Munari et al. (1992). We then used the analytic expression of Bessell \& Wood (1984), involving that color, to obtain a bolometric correction at $K$. This, combined with our value of $M_{\mathrm{bol}}$ from the $\mathrm{M}$ giant's minimum radius, produces $M_{K}=-6.54 \mathrm{mag}$ and results in a minimum distance of $4.0 \mathrm{kpc}$ if reddening is ignored. Including an extinction value of 0.5 mag decreases the distance to $3.2 \mathrm{kpc}$. These distances are somewhat greater than the $2.3 \mathrm{kpc}$ estimate of Mikołajewska et al. (1997). The galactic latitude places the star $9^{\circ}$ below the Galactic plane, while its longitude is within $16^{\circ}$ of the Galactic center. Thus, the distance and position of AE Ara toward the center of the Milky Way are consistent with its membership in an older stellar population.

From our orbital solution the ephemeris for conjunctions with the $\mathrm{M}$ giant in front, which corresponds to times of mid-eclipse, is

$$
T_{\text {conj }}(\mathrm{HJD})=2,453,448.7( \pm 10)+803( \pm 9) E,
$$

where $E$ is an integer number of cycles.

The above ephemeris is for the eclipse of the putative white dwarf by the M giant. The tiny size of the white dwarf and its luminosity at visual wavelengths means that an eclipse of the white dwarf would result in a change of less than $0.01 \mathrm{mag}$. However, an eclipse of the extended hot disk (e.g., Hutchings et al. 1983) would be easily observed at optical wavelengths.

Mikołajewska et al. (2003) examined an extensive series of visual observations obtained from 1986 through 1997 and concluded that the roughly sinusoidal light curve variations are associated with the orbital period. Skopal et al. (2007) added more recent visual observations to that data set and reported that the best-defined new minimum agrees with the ephemeris of Mikołajewska et al. (2003). Our orbital ephemeris also is consistent, within its errors, with that best new minimum of JD $2,453,474 \pm 20$ (Skopal et al. 2007). Despite the identification of light variations that have the same periodicity as the radial velocity orbit, neither Mikołajewska et al. (2003) nor Skopal et al. (2007) posited that eclipses occur. In addition to the visual observations, AE Ara has been observed as part of the All Sky Automated Survey (ASAS) program (Pojmanski 2002). The photometric database ${ }^{5}$ from that survey contains about $550 \mathrm{~V}$ mag observations, obtained from 2001 to the present. Those observations show that the $V$ amplitude of the current cycle is greater than $2 \mathrm{mag}$, the system being slightly brighter at maximum than in the previous cycle noted by Skopal et al. (2007). Again, no eclipses are obvious in the data. The lack of eclipses is consistent with our adopted component masses, which result in an orbital inclination of $51^{\circ}$.

\section{6. $\mathrm{Y}$ CRA $=$ HD $166813=$ SS $73-144=$ HEN 3-1632= HV 169}

\subsection{Short History}

By the beginning of the 20th century Harvard College Observatory was the epicenter of most variable star observation and analysis (Mayall 1976). In one of the early publications resulting from that work, Pickering et al. (1901) provided a list of 64 new variable stars, many of which were first identified because they had hydrogen emission lines in their spectra on the Harvard College Observatory plates. According to that report, Fleming discovered the large brightness variations of one star, which was later designated HV 169. More than a decade later, having been assigned the variable star name Y CrA, Fleming (1912) listed it in a table of stars with peculiar spectra, where it was characterized as having brightness changes ranging from 12.0 to $12.9 \mathrm{mag}$ and an irregular period.

The emission lines in its spectrum were bright enough so that it was given an HD number. Its spectrum was characterized as Md (Pickering et al. 1901), which in the notation of the Henry Draper Catalog means that the spectrum was like that of Mira at minimum except that the hydrogen emission features of Y CrA were similar in strength to Mira at maximum light. Cannon \& Pickering (1916) described its spectrum as showing emission lines of $\mathrm{H} \beta, \mathrm{H} \gamma$, and $\mathrm{H} \delta$ plus one bright band at about $4653 \AA$. In Table 5 of his catalog of emission line stars Bidelman (1954) listed Y CrA in a group of stars with combination spectra. Those objects show high-excitation emission lines and low-temperature absorption lines, and the vast majority are now identified as symbiotic stars. Using moderately deep, lowdispersion objective-prism spectra, Sanduleak \& Stephenson (1973) surveyed the southern Milky Way to search for luminous and emission-lined stars. They classified star number 144 in their 1973 list as a Z Andromodae-like star and thus, a

\footnotetext{
5 http://www.astrouw.edu.pl/asas/
} 
Table 5

Absorption Line Radial Velocities of Y CRA

\begin{tabular}{ccccc}
\hline \hline $\begin{array}{c}\text { HJD } \\
(2400000+)\end{array}$ & Phase & $\begin{array}{c}\text { Velocity } \\
\left(\mathrm{km} \mathrm{s}^{-1}\right)\end{array}$ & $\begin{array}{c}O-C \\
\left(\mathrm{~km} \mathrm{~s}^{-1}\right)\end{array}$ & Observatory $^{\mathrm{a}}$ \\
\hline 52047.284 & 0.716 & -50.9 & -0.4 & MSO \\
52095.317 & 0.745 & -53.1 & -1.7 & MSO \\
52131.170 & 0.767 & -51.7 & 0.4 & MSO \\
52358.298 & 0.908 & -56.0 & -0.7 & MSO \\
52402.100 & 0.935 & -54.8 & 0.5 & MSO \\
52449.102 & 0.964 & -54.2 & 0.8 & MSO \\
52504.045 & 0.998 & -56.8 & -2.6 & MSO \\
52749.877 & 0.150 & -47.7 & 0.2 & Gemini S \\
53130.956 & 0.385 & -44.5 & -0.2 & KPNO \\
53179.845 & 0.415 & -43.0 & 1.5 & KPNO \\
53538.851 & 0.637 & -48.0 & 0.3 & KPNO \\
53900.873 & 0.860 & -53.1 & 1.5 & KPNO \\
54231.966 & 0.065 & -49.8 & 1.8 & KPNO \\
54270.856 & 0.089 & -50.8 & -0.3 & KPNO \\
54634.863 & 0.314 & -45.8 & -1.4 & KPNO \\
54956.946 & 0.513 & -45.9 & -0.3 & KPNO \\
54974.902 & 0.524 & -46.0 & -0.2 & CTIO \\
54998.857 & 0.539 & -45.8 & 0.3 & KPNO \\
55036.727 & 0.562 & -46.1 & 0.5 & CTIO \\
55043.769 & 0.566 & -46.8 & -0.1 & Gemini S \\
\hline
\end{tabular}

Notes.

a MSO, Mount Stromlo Observatory; Gemini S, Gemini South Observatory; KPNO, Kitt Peak National Observatory; CTIO, Cerro Tololo Inter-American Observatory.

likely symbiotic. In addition to hydrogen emission its spectrum contained bright $\mathrm{O}$ III lines as well as a $\mathrm{He}$ II emission line at $4686 \AA$ that was stronger than that of $\mathrm{H} \beta$. From plates taken a quarter century earlier, Henize (1976) provided an extensive catalog of southern stars with emission at $\mathrm{H} \alpha$, identifying number 1632 in his list as Y CrA.

Following up on the work of Sanduleak \& Stephenson (1973), Allen (1978) acquired a low-dispersion slit spectrum of Y CrA. He identified it as a reddened symbiotic star, stating that it had strong $\mathrm{TiO}$ bands and that moderate strength forbidden $\mathrm{Fe}$ VI and Fe vII emission lines were visible.

The various classifications of the $\mathrm{Y}$ CrA spectrum are in excellent accord. Allen (1980) called its spectral class M5 from a spectrum at $2 \mu \mathrm{m}$. Medina Tanco \& Steiner (1995) used the strength of absorption bands in the near-infrared to deduce a spectral class of M6. Similarly, Gutierrez-Moreno et al. (1999) classified it as M6.1 and Mürset \& Schmid (1999) gave it an M6 classification.

Y CrA was included in the symbiotic star catalog of Allen (1984), where a low-dispersion spectrum was shown, as well as the more recent catalog of Belczyński et al. (2000). No orbit for the system was available at that time.

\subsection{Spectroscopic Orbit}

From 2001 May to 2009 July we acquired 20 radial velocities of Y CrA (Table 5). An analysis of these velocities, which cover 1.8 orbital cycles, produced an initial period of 1595 days. Adopting this period, we obtained preliminary orbital elements, which were then refined resulting in a period of 1619 days. Because of the relatively low orbital eccentricity of $0.17 \pm$ 0.09 , we determined a circular-orbit solution, which has a period of 1598 days. Although the tests of Lucy \& Sweeney (1971) indicated that the circular orbit is to be preferred, we have instead adopted the eccentric orbit solution in Table 6

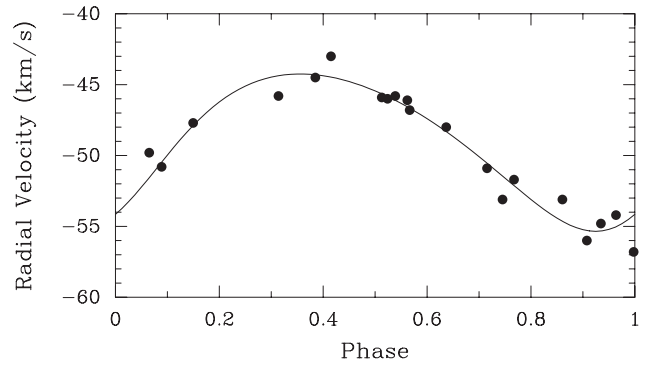

Figure 6. M giant radial velocities of $\mathrm{Y}$ CrA (filled circles) compared with its computed eccentric orbit (solid line). Zero phase is a time of periastron passage.

Table 6

Orbital Elements and Related Parameters of Y CRA

\begin{tabular}{lc}
\hline \multicolumn{1}{c}{ Parameter } & Value \\
\hline$P($ days $)$ & $1619.0 \pm 35.8$ \\
$T(\mathrm{HJD})$ & $2454127 \pm 124$ \\
$\gamma\left(\mathrm{km} \mathrm{s}^{-1}\right)$ & $-49.04 \pm 0.31$ \\
$K\left(\mathrm{~km} \mathrm{~s}^{-1}\right)$ & $5.55 \pm 0.43$ \\
$e$ & $0.173 \pm 0.088$ \\
$\omega(\mathrm{deg})$ & $218.3 \pm 28.5$ \\
$a \sin i\left(10^{6} \mathrm{~km}\right)$ & $121.7 \pm 10.1$ \\
$f(m)\left(M_{\odot}\right)$ & $0.0275 \pm 0.0066$ \\
Standard error of an observation & \\
of unit weight $\left(\mathrm{km} \mathrm{s}^{-1}\right)$ & 1.2 \\
\hline
\end{tabular}

for two reasons. First, like Barium stars, symbiotic stars are mass transfer binaries, generally consisting of a late-type giant star and a white dwarf companion. Verbunt \& Phinney (1995) have shown that enhanced tidal interactions, due to the stellar evolution of such systems, lead to circularization of their orbits. Fekel et al. (2007) examined the period-eccentricity distribution of S-type symbiotics. They found that over $80 \%$ of the systems with periods less than 800 days have circular orbits, while the percentage drops to $22 \%$ for symbiotics with periods greater than 800 days. Because the orbital period of $\mathrm{Y} \mathrm{CrA}$ is twice as long as the longest period at which most symbiotics have circular orbits, its orbit is likely eccentric. Second, although the uncertainty is a significant fraction of the eccentricity value, the eccentricity itself is moderately large, being nearly 0.2 . From the orbital solution the standard error of an observation of unit weight is equal to $1.2 \mathrm{~km} \mathrm{~s}^{-1}$, at least twice as large as our symbiotic orbits with the smallest velocity residuals, and so part of the velocity variations results from pulsation (Lebzelter \& Hinkle 2002). Figure 6 compares our velocities to the computed orbit.

\subsection{Emission Line Spectrum}

We examined our nine spectra of Y CrA that were obtained in the $1 \mu \mathrm{m}$ region, where several emission features occur in most symbiotics. All but one of these spectra show the Paschen $\delta$ line as a rather weak emission feature, being similar in strength or weaker than the strongest absorption features in this region. In the one exception of 2008 June, Paschen $\delta$ emission above the continuum level is absent although the absorption feature is completely filled in by emission. A second emission line typically seen in this wavelength region, He II at $1.0123 \mu \mathrm{m}$, is absent in all but the two spectra obtained in 2009. Because of the weakness of the two emission features we have not attempted to measure velocities for either one. 


\subsection{Pulsation Period Search}

The ASAS program (Pojmanski 2002), which nightly monitors the southern hemisphere sky, has obtained over 600 photometric observations of Y CrA. The entry for that star in the web-based ASAS Catalog of Variable Stars gives a period of 84.27 days and a $V$ amplitude of 0.4 mag. However, the phase diagram for that period shows that the points have a huge scatter.

To see whether such a period might be detected in the radial velocities, the velocity residuals to the adopted eccentric orbit were searched for periods between 10 and 1000 days. We found no evidence of a periodicity at 84.27 days. We examined the phase curves of two best periods, 16.3765 and 15.6675 days, that were found in our velocity residuals, but neither showed a convincing periodicity.

\subsection{Discussion}

Munari et al. (1992) observed Y CrA as part of an optical and infrared photometric survey of symbiotic stars, but that star was one of a small number for which they only obtained optical magnitudes. More recently, Phillips (2007) provided infrared magnitudes for Y CrA from the Two Micron All Sky Survey (2MASS) survey. The various classifications of the Y CrA spectrum range from M5-M6.1, and we adopt the M6 classification of Mürset \& Schmid (1999).

The spectroscopically derived information for $\mathrm{Y} \mathrm{CrA}$ is more limited than that for AE Ara. The single-lined binary orbit only provides a value for the mass function, $f(m)$, which from Table 6 is $0.027 M_{\odot}$, nearly identical to that for AE Ara. The mass function can be written as

$$
f(m)=\left(m_{\mathrm{wd}}^{3} \sin ^{3} i\right) /\left(m_{\mathrm{rg}}+m_{\mathrm{wd}}\right)^{2} .
$$

Therefore, its value depends on $m_{\mathrm{wd}}$, the mass of the presumed white dwarf, $m_{\mathrm{rg}}$, the mass of the red giant, and $i$, the orbital inclination. Adopting a mass of $1.6 M_{\odot}$ for the red giant, as we did for AE Ara, produces a minimum mass of $0.49 M_{\odot}$ for the hot companion, which increases to $0.6 M_{\odot}$ if the inclination is decreased from $90^{\circ}$ to $55^{\circ}$. Thus, an orbit with the latter inclination reproduces the masses adopted for AE Ara. As discussed below, Y CrA is likely a member of the old disk population. According to Wallerstein (1981), old disk stars should have masses of $1.0-1.5 M_{\odot}$, somewhat less than the value of $1.6 M_{\odot}$. If the adopted mass for the red giant is reduced, for a fixed value of the inclination the corresponding mass of the hot companion is also reduced. For example, a $1.2 M_{\odot}$ produces a typical $0.6 M_{\odot}$ white dwarf for an inclination of $31^{\circ}$. Thus, the likely masses for this system produce low orbital inclinations, arguing that the system does not eclipse.

We have not determined a $v \sin i$ value for $\mathrm{Y} C r A$, nor is there one available in the literature. The Roche lobe radius of the giant depends on the separation of the stars and their mass ratio. However, we have adopted an eccentric orbit for Y CrA, so its separation will vary. Nevertheless, the eccentricity is not extremely large, and we can get an estimate of the minimum effective Roche lobe radius by adopting the smaller periastron separation rather than the semimajor axis. If we assume masses of 1.6 and $0.6 M_{\odot}$, Equation (2) of Eggleton (1983) produces a minimum Roche-lobe radius of $292 R_{\odot}$ for the M giant. Of course, as the separation of the stars increases the size of the M giant's Roche lobe will also increase. According to Dumm \& Schild (1998), the median radius of an M6 III is $152 R_{\odot}$, while van Belle et al. (1999) find an average radius for that spectral type of $148 R_{\odot}$. The minimum Roche lobe radius for $\mathrm{Y} \mathrm{CrA}$ is nearly twice as large as those values, so it is very unlikely that the M giant of Y CrA fills its Roche lobe.

From our adopted spectral class of M6 (Mürset \& Schmid 1999) we assume an effective temperature of $3380 \mathrm{~K}$ (Dyck et al. 1996) for Y CrA. That value and our adopted radius result in a luminosity of $2630 L_{\odot}$, leading to $M_{\text {bol }}=-3.8 \mathrm{mag}$. Comparison with the theoretical predictions of Vassiliadis \& Wood (1993) indicates that the $M$ giant is likely an AGB star. According to Seaquist et al. (1993), the mass loss rate is $3.4 \times 10^{-7} M_{\odot} \mathrm{yr}^{-1}$.

To estimate the distance to Y CrA, we adopted its $K$ mag and $J-K$ color from the 2MASS infrared survey (Phillips 2007). Although the 2MASS $K$ magnitude passband is not exactly that of the Johnson magnitude system, we have used it because there is no $K$ mag from Munari et al. (1992). We then used the analytic expression of Bessell \& Wood (1984), involving that color, to obtain a bolometric correction at $K$. This, combined with our value of $M_{\mathrm{bol}}$, produces $M_{K}=-6.65 \mathrm{mag}$ and results in a distance of $4.2 \mathrm{kpc}$ if reddening is ignored. Including an extinction value of 0.5 mag decreases the distance to $3.4 \mathrm{kpc}$. The galactic latitude places the star $12^{\circ}$ below the Galactic plane, while its longitude is within $10^{\circ}$ of the Galactic center. Our center-of-mass velocity for $\mathrm{Y} \mathrm{CrA}$ is $-49 \mathrm{~km} \mathrm{~s}^{-1}$. Thus, the distance and position of Y CrA toward the center of the Galaxy and its rather large systemic velocity argue that it, like most symbiotics, belongs to the old disk population (Wallerstein 1981).

The median period for S-type symbiotic stars with spectroscopic orbits is 720 days or nearly $2 \mathrm{yr}$. The orbital period of Y CrA, 1619 days, is the second longest of any S-type system for which a spectroscopic orbit so far has been determined. The longest period, 5689 days, belongs to $\mathrm{CH}$ Cyg. Although classified as an S-type symbiotic, $\mathrm{CH}$ Cyg has several properties similar to D-type symbiotics (Hinkle et al. 2009), which have much longer periods than the S-type systems.

From our orbital elements the ephemeris for conjunctions with the $\mathrm{M}$ giant in front, which corresponds to times of mideclipse if the binary has a high enough inclination, is

$$
T_{\text {conj }}(\mathrm{HJD})=2,454,126( \pm 124)+1619( \pm 36) E,
$$

where $E$ represents an integer number of cycles.

\section{SS $73-147=$ HEN 2-374}

\subsection{Short History}

Compared to AE Ara and Y CrA, the discovery of SS 73147 as a star of particular interest occurred much more recently. The (1966) conducted a survey to find $\mathrm{H} \alpha$ emission line objects associated with regions of irregular dark nebulosities. The star listed as THA 34-33 was found to have strong $\mathrm{H} \alpha$ emission. In his objective prism survey of the southern Milky Way, made to search for planetary nebulae, Henize (1967) listed the star as number 374 . Having carried out a somewhat similar survey, Sanduleak \& Stephenson (1973) suspected that star number 147 in their list was a possible $\mathrm{Z}$ Andromedae-like star and might have TiO absorption. Allen (1978) followed up on their work and obtained a low-dispersion spectrum of the star that clearly showed prominent $\mathrm{TiO}$ bands. Weak emission features include the He II line at $4686 \AA$ and forbidden Ne v.

As with AE Ara and Y CrA, several groups have classified the spectrum of SS 73-147. Medina Tanco \& Steiner (1995) 


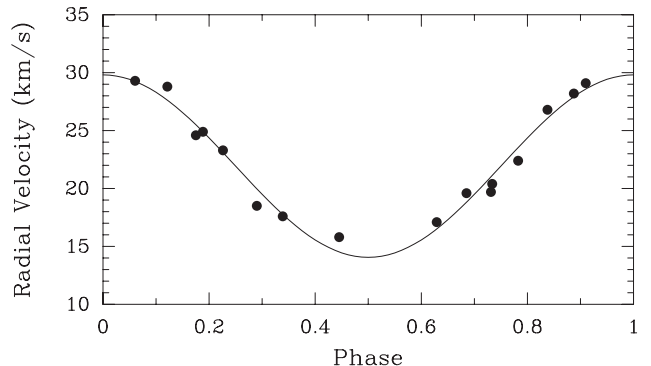

Figure 7. M giant radial velocities of SS 73-147 (filled circles) compared with its computed circular orbit (solid line). Zero phase is a time of maximum velocity.

Table 7

Absorption Line Radial Velocities of SS 73-147

\begin{tabular}{lcccc}
\hline \hline $\begin{array}{c}\text { HJD } \\
(2400000+)\end{array}$ & Phase & $\begin{array}{c}\text { Velocity } \\
\left(\mathrm{km} \mathrm{s}^{-1}\right)\end{array}$ & $\begin{array}{c}O-C \\
\left(\mathrm{~km} \mathrm{~s}^{-1}\right)\end{array}$ & Observatory $^{\mathrm{a}}$ \\
\hline 52048.224 & 0.629 & 17.1 & 0.6 & MSO \\
52094.267 & 0.685 & 19.6 & 0.8 & MSO \\
52134.099 & 0.734 & 20.4 & -0.7 & MSO \\
52402.202 & 0.061 & 29.3 & 0.1 & MSO \\
52452.115 & 0.121 & 28.8 & 1.2 & MSO \\
52507.117 & 0.188 & 24.9 & 0.0 & MSO \\
53098.880 & 0.910 & 29.1 & 0.5 & Gemini S \\
53537.929 & 0.445 & 15.8 & 1.3 & KPNO \\
53860.001 & 0.838 & 26.8 & 0.7 & KPNO \\
53900.903 & 0.888 & 28.2 & 0.3 & KPNO \\
54230.968 & 0.290 & 18.5 & -1.5 & KPNO \\
54270.891 & 0.339 & 17.6 & -0.2 & KPNO \\
54592.960 & 0.731 & 19.7 & -1.3 & KPNO \\
54634.894 & 0.782 & 22.4 & -1.1 & KPNO \\
54956.907 & 0.175 & 24.6 & -0.9 & KPNO \\
54998.893 & 0.226 & 23.3 & 0.2 & KPNO \\
\hline
\end{tabular}

Notes.

a MSO, Mount Stromlo Observatory; Gemini S, Gemini South Observatory; KPNO, Kitt Peak National Observatory.

concluded that the red giant has a spectral class of M5, while Mikołajewska et al. (1997) estimated M4, and Mürset \& Schmid (1999) found M5.5.

\subsection{Spectroscopic Orbit}

From 2001 May to 2009 June we acquired 16 radial velocities of SS 73-147 (Table 7). An analysis of those velocities produced an initial period of 821 days, so our observations span 3.5 orbital cycles. Adopting this period, we obtained preliminary orbital elements, which were then slightly improved. Because that solution has a low orbital eccentricity of $0.11 \pm 0.05$, we also determined a circular-orbit solution. The tests of Lucy \& Sweeney (1971) indicated that the circular orbit is to be preferred. The standard error of an individual velocity is $1.0 \mathrm{~km} \mathrm{~s}^{-1}$. This is smaller than for the orbits of our other two stars, but the value is still twice as large as the values for some of our previously determined orbits, suggesting that a portion of the velocity residuals results from pulsation. With a period close to 800 days, we have chosen the circular solution, which is listed in Table 8 . Because a time of periastron passage, $T$, is undefined for a circular orbit, as recommended by Batten et al. (1989), we have instead listed $T_{0}$, a time of maximum velocity, in the orbital elements table. Figure 7 compares the velocities to the computed orbit.
Table 8

Orbital Elements and Related Parameters of SS 73-147

\begin{tabular}{lc}
\hline \hline \multicolumn{1}{c}{ Parameter } & Value \\
\hline$P$ (days) & $820.3 \pm 4.1$ \\
$T_{0}(\mathrm{HJD})$ & $2453172.8 \pm 5.6$ \\
$\gamma\left(\mathrm{km} \mathrm{s}^{-1}\right)$ & $21.93 \pm 0.26$ \\
$K\left(\mathrm{~km} \mathrm{~s}^{-1}\right)$ & $7.88 \pm 0.47$ \\
$e$ & 0.0 (adopted) \\
$a \sin i\left(10^{6} \mathrm{~km}\right)$ & $88.85 \pm 5.36$ \\
$f(m)\left(M_{\odot}\right)$ & $0.0416 \pm 0.0075$ \\
Standard error of an observation & \\
of unit weight $\left(\mathrm{km} \mathrm{s}^{-1}\right)$ & 1.0
\end{tabular}

Table 9

Emission Line Radial Velocities of SS 73-147

\begin{tabular}{cccc}
\hline \hline $\begin{array}{c}\text { HJD } \\
(2400000+)\end{array}$ & Phase & $\begin{array}{c}\text { H I } \\
\text { Velocity } \\
\left(\mathrm{km} \mathrm{s}^{-1}\right)\end{array}$ & $\begin{array}{r}\text { He II } \\
\text { Velocity } \\
\left(\mathrm{km} \mathrm{s}^{-1}\right)\end{array}$ \\
\hline 53537.929 & 0.445 & 16.3 & 17.5 \\
53860.001 & 0.838 & 27.3 & 28.8 \\
53900.903 & 0.888 & 24.0 & 23.5 \\
54230.968 & $0.290^{\mathrm{a}}$ & $\ldots$ & 8.5 \\
54270.891 & 0.339 & $42.9^{\mathrm{b}}$ & 12.7 \\
54592.960 & 0.731 & 29.5 & 44.3 \\
54634.894 & 0.782 & 29.4 & 34.1 \\
54956.907 & $0.175^{\mathrm{a}}$ & $\ldots$ & 0.4 \\
54998.893 & $0.226^{\mathrm{a}}$ & $\ldots$ & 5.1 \\
\hline
\end{tabular}

Notes.

a The Paschen $\delta$ line is not in emission above the continuum at this phase.

b The Paschen $\delta$ emission line is very weak and poorly defined.

\subsection{Emission Line Spectrum}

Having determined the orbit for the M giant of SS 73-147, we next examined the emission lines near $1 \mu \mathrm{m}$ that are found in our KPNO spectra. For SS 73-147 only Paschen $\delta$ and He II are typically in emission, and their profiles are usually reasonably symmetric. The radial velocities of the two emission features are listed in Table 9, as well as the phases of those velocities in the $\mathrm{M}$ giant orbit. The latter indicate that the hydrogen emission above the continuum disappears when the hot component is near superior conjunction.

Mikołajewska et al. (1989) stated that in symbiotics the He II emission lines should follow the hot component. Thus, we obtained a circular orbit solution for the He II line, adopting our orbital period of the $\mathrm{M}$ giant and solving for the other orbital elements. Like AE Ara, which has a similar period, the phases of the emission velocities have a limited distribution and do not cover velocity maximum or minimum. The center-of-mass velocity for that orbit differs from the systemic velocity of the $\mathrm{M}$ giant orbit by less than $1 \mathrm{~km} \mathrm{~s}^{-1}$. Using the absorption and emission velocity semi-amplitudes, the mass ratio for the red giant and putative white dwarf, $M_{\mathrm{rg}} / M_{\mathrm{wd}}$, is 2.4 , in reasonable accord with the expectation for symbiotics (Mikołajewska 2003). However, Figure 8, which is a plot of the He II velocities phased with the $M$ giant orbit, clearly shows that the emission line velocities are not in antiphase. Instead they differ from that expected orientation by $\sim 0.18$ phase units. Thus, although the emission appears to be generally associated with the companion of the M giant, the He II emission line velocities of SS 73-147 are not necessarily a good proxy for the hot component. The limited observed phases for those emission velocities suggest that region where the He II line originates lags in phase behind the presumed hot companion. 


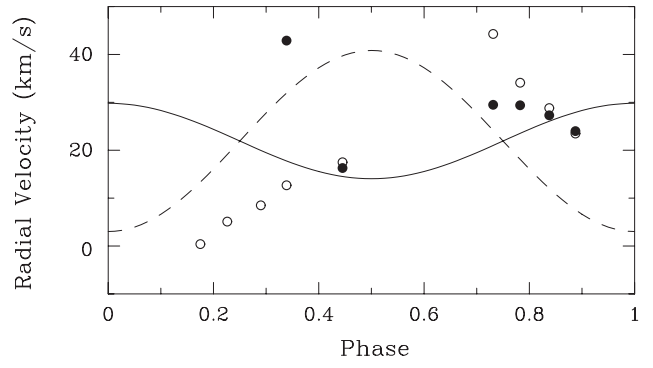

Figure 8. Emission line radial velocities of SS 73-147 (filled circles = H I, open circles = He II) compared to the orbit determined for the $\mathrm{M}$ giant (solid line). The dashed line is the predicted orbit for a secondary with an adopted mass ratio of 2.4. Zero phase is a time of maximum velocity of the $\mathrm{M}$ giant.

\subsection{Pulsation Period Search}

The velocity residuals to the adopted circular orbit were searched for periods between 10 and 1000 days. The best period of 36.08 days produced a velocity curve that was clearly asymmetric in shape. Once again the phase curve of the velocity residuals was not particularly convincing.

\subsection{Discussion}

In a survey of symbiotic stars that was designed to determine the evolutionary status of the red giant components, Kenyon \& Gallagher (1983) obtained near-infrared magnitudes of SS 73147 that are similar in value to those of the more recent 2MASS survey (Skrutskie et al. 2006). The $V$ mag observations from the photometric database of the ASAS program (Pojmanski 2002), obtained from 2001 to the present, show only modest variability with no clear evidence of periodicity or eclipses. The average $V$ mag is $\sim 13.5$.

The three spectral classifications of SS 73-147 are reasonably consistent, ranging from M4 (Mikołajewska et al. 1997) to M5.5 (Mürset \& Schmid 1999). We adopt the latter value in this work. From a low-resolution spectrogram, Mikołajewska et al. (1997) provided a limited analysis of SS 73-147. They estimated a system distance of $2.5 \mathrm{kpc}$ and a hot component temperature between 75,000 and $100,000 \mathrm{~K}$ plus a luminosity of $1300 L_{\odot}$.

As was the case for Y CrA, we do not have a reliable mass ratio to help restrict the masses and must resort to the mass function instead. The value of the mass function for SS 73-147 is $0.042 M_{\odot}$, somewhat larger than that for AE Ara and Y Cra. Without a suitable orbit for the secondary, we must make several assumptions to explore the status of the system. If we again adopt a mass of $1.6 M_{\odot}$ for the primary, then the mass of the hot component is $0.58 M_{\odot}$ for an inclination of $90^{\circ}$ and increases to $0.67 M_{\odot}$ when the inclination decreases to $60^{\circ}$. If a smaller red giant mass is adopted, for a given inclination the mass of the secondary also decreases. Since there is no clear evidence for eclipses, we adopt the above masses for an inclination of $60^{\circ}$.

With the same procedure that we used for AE Ara, from a single spectrum we determined $v \sin i=6 \pm 2 \mathrm{~km} \mathrm{~s}^{-1}$ for the $M$ giant component of SS 73-147. Zamanov et al. (2007) used two different methods to also measure its $v \sin i$. The average of the three values is $7 \mathrm{~km} \mathrm{~s}^{-1}$.

Following Schmutz et al. (1994), if we assume that the giant is synchronously rotating, the minimum radius is $113.5 R_{\odot}$. If the rotational inclination is reduced from $90^{\circ}$ to $60^{\circ}$, the radius is increased to $131 R_{\odot}$.

From our adopted spectral class of M5.5 (Mürset \& Schmid 1999) we assume an effective temperature of 3425 K (Dyck et al. 1996). That, combined with our adopted radius, results in a luminosity of $1547 L_{\odot}$, leading to $M_{\text {bol }}=-3.6 \mathrm{mag}$.

To estimate the minimum distance to SS 73-147, we adopted its $K$ mag and $J-K$ color from Kenyon \& Gallagher (1983). We then used the analytic expression of Bessell \& Wood (1984), to obtain a bolometric correction at $K$. This, combined with our value of $M_{\mathrm{bol}}$ from the $\mathrm{M}$ giant's adopted radius, produces $M_{K}=-6.44 \mathrm{mag}$ and results in a minimum distance of $4.0 \mathrm{kpc}$ if reddening is ignored. Including an extinction value of $0.5 \mathrm{mag}$ decreases the distance to $3.1 \mathrm{kpc}$. These values are somewhat larger than the $2.5 \mathrm{kpc}$ value estimated by Mikołajewska et al. (1997). At $22 \mathrm{~km} \mathrm{~s}^{-1}$ the systemic velocity is moderate but not nearly as large as that for $\mathrm{Y} \mathrm{CrA}$. The galactic latitude places the star $2^{\circ}$ below the Galactic plane, while its longitude is within $10^{\circ}$ of the Galactic center. Thus, the distance and position of SS 73-147 toward the center of the Milky Way are consistent with its membership in an older stellar population.

Assuming synchronous rotation and a radius for the red giant, Zamanov et al. (2007) also computed an upper limit of 1091 days for the orbital period of SS 73-147. Our observed orbital period of 820 days is less than their estimated upper limit, and so it is consistent with their prediction.

From our orbital elements the ephemeris for conjunctions with the M giant in front, which corresponds to times of mideclipse if the binary has a high enough inclination, is

$$
T_{\text {conj }}(\mathrm{HJD})=2,453,173( \pm 6)+820( \pm 4) E,
$$

where $E$ represents an integer number of cycles.

We thank NOAO for enabling several aspects of this research, including support of the KPNO coudé feed telescope, access to time on the Gemini and SMARTS telescopes, and travel support for K. Hinkle and R. Joyce. This paper is based in part on observations obtained at the Gemini Observatory, which is operated by the Association of Universities for Research in Astronomy, Inc., under a cooperative agreement with the NSF on behalf of the Gemini partnership: the National Science Foundation (US), the Particle Physics and Astronomy Research Council (UK), the National Research Council (Canada), CONICYT (Chile), the Australian Research Council (Australia), CNPq (Brazil), and CONICRT (Argentina). The observations were obtained with the Phoenix infrared spectrograph, which was developed and is operated by the National Optical Astronomy Observatory. The Gemini/Phoenix spectra were obtained as part of programs GS2003A-DD-1, GS-2003B-DD-1, and GS-2004A-DD-1. Observations were also obtained at the Gemini South Observatory as part of Poor Weather Queue program no. GS-2009A-Q-88. Several additional observations were acquired through the NOAO share of time on one of the SMARTS consortium telescopes. This research has been supported in part by NASA grants NCC5511 and NSF grant HRD-9706268 to Tennessee State University. We have made use of the SIMBAD database, operated by CDS in Strasbourg, France, as well as NASA's Astrophysics Data System Abstract Service.

\section{REFERENCES}

Allen, D. A. 1978, MNRAS, 184, 601

Allen, D. A. 1980, MNRAS, 192, 521

Allen, D. A. 1984, PASA, 5, 369

Allen, D. A., \& Glass, I. S. 1974, MNRAS, 167, 337

Andrillat, Y., Jaschek, M., \& Jaschek, C. 1990, A\&AS, 85, 855

Batten, A. H., Fletcher, J. M., \& MacCarthy, D. G. 1989, Publ. Dominion Astrophys. Obs. Victoria, 17, 1 
Belczyński, K., Mikołajewska, J., Munari, U., Ivison, R. J., \& Friedjung, M. 2000, A\&AS, 146, 407

Bessell, M. S., \& Wood, P. R. 1984, PASP, 96, 247

Bidelman, W. P. 1954, ApJS, 1, 175

Cannon, A. J. 1933, Harvard Obs. Bull., 891, 9

Cannon, A. J., \& Pickering, E. C. 1916, Ann. Harvard College Obs., 76, 19

Dobrzycka, D., Kenyon, S. J., \& Mikołjewska, J. 1993, AJ, 106, 284

Dumm, T., \& Schild, H. 1998, New Astron., 3, 137

Dyck, H. M., Benson, J. A., van Belle, G. T., \& Ridgway, S. T. 1996, AJ, 111, 1705

Eggleton, P. P. 1983, ApJ, 269, 368

Fekel, F. C. 1997, PASP, 109, 514

Fekel, F. C., Hinkle, K. H., Joyce, R. R., \& Skrutskie, M. F. 2000a, AJ, 120, 3255

Fekel, F. C., Hinkle, K. H., Joyce, R. R., \& Skrutskie, M. F. 2001, AJ, 121, 2219

Fekel, F. C., Hinkle, K. H., Joyce, R. R., Wood, P. R., \& Howarth, I. D. 2008, AJ, 136, 146

Fekel, F. C., Hinkle, K. H., Joyce, R. R., Wood, P. R., \& Lebzelter, T. 2007, AJ, 133,17

Fekel, F. C., Joyce, R. R., Hinkle, K. H., \& Skrutskie, M. F. 2000b, AJ, 119, 1375

Fitzpatrick, M. J. 1993, in ASP Conf. Ser. 52, Astronomical Data Analysis Software and Systems II, ed. R. J. Hanish, R. V. J. Brissenden, \& J. Barnes (San Francisco, CA: ASP), 472

Fleming, W. P. 1912, Ann. Harvard College Obs., 56, 165

Garcia, M. R. 1986, AJ, 91, 1400

Gutierrez-Moreno, A., Moreno, H., \& Costa, E. 1999, PASP, 111, 571

Henize, K. G. 1967, ApJS, 14, 125

Henize, K. G. 1976, ApJS, 30, 491

Hinkle, K. H., Fekel, F. C., Joyce, R. R., Wood, P. R., Smith, V., \& Lebzelter, T. 2006, AJ, 641, 479

Hinkle, K. H., Fekel, F. C., \& Joyce, R. R. 2009, ApJ, 692, 1360

Hinkle, K. H., et al. 1998, Proc. SPIE, 3354, 810

Hutchings, J. B., Cowley, A. P., Ake, T. B., \& Imhoff, C. L. 1983, ApJ, 275, 271

Johansson, S. 1977, MNRAS, 178, 17p

Joyce, R. R. 1992, in ASP Conf. Ser. 23, Astronomical CCD Observing and Reduction Techniques, ed. S. Howell (San Francisco, CA: ASP), 258

Joyce, R. R., Hinkle, K. H., Meyer, M. R., \& Skrutskie, M. F. 1998, Proc. SPIE, 3354, 741

Keenan, P. C., \& McNeil, R. C. 1989, ApJS, 71, 245

Kenyon, S. J., \& Gallagher, J. S. 1983, AJ, 88, 666

Kenyon, S. J., \& Webbink, R. F. 1984, ApJ, 279, 252

Lebzelter, T., \& Hinkle, K. H. 2002, A\&A, 393, 563

Lucy, L. B., \& Sweeney, M. A. 1971, AJ, 76, 544
Mayall, M. W. 1976, J. Am. Assoc. Var. Star Obs., 5, 7

Medina Tanco, G. A., \& Steiner, J. E. 1995, AJ, 109, 1770

Merrill, P. W., \& Burwell, C. G. 1943, ApJ, 98, 153

Mikołajewska, J. 2003, in Symbiotic Stars Probing Stellar Evolution, ed. R. L. M. Corradi, J. Mikołajewska, \& T. J. Mahoney (San Francisco, CA: ASP), 9

Mikołajewska, J., Acker, A., \& Stenholm, B. 1997, A\&A, 327, 191

Mikołajewska, J., Kenyon, S. J., \& Mikołajewski, M. 1989, AJ, 98, 1427

Mikołajewska, J., Quiroga, C., Brandi, E., Garcia, L. G., Ferrer, O. E., \& Belczynski, K. 2003, in Symbiotic Stars Probing Stellar Evolution, ed. R. L. M. Corradi, J. Mikołajewska, \& T. J. Mahoney (San Francisco, CA: ASP), 147

Munari, U., Yudin, B. F., Taranova, O. G., Massone, G., Marang, F., Roberts, G., Winkler, H., \& Whitelock, P. A. 1992, A\&AS, 93, 383

Mürset, U., Dumm, T., Isenegger, S., Nussbaumer, H., Schild, H., Schmid, H. M., \& Schmutz, W. 2000, A\&A, 353, 952

Mürset, U., Nussbaumer, H., Schmid, H. M., \& Vogel, M. 1991, A\&A, 248, 458

Mürset, U., \& Schmid, H. M. 1999, A\&AS, 137, 473

Peimbert, M., \& Costero, R. 1961, Bol. Obs. Tonantzintla Tacubaya, 3, 33

Percy, J. R., Wilson, J. B., \& Henry, G. W. 2001, PASP, 113, 983

Phillips, J. P. 2007, MNRAS, 376, 1120

Pickering, E. C., Colson, H. R., Fleming, W. P., \& Wells, L. D. 1901, ApJ, 13, 226

Pojmanski, G. 2002, Acta Astron., 52, 397

Russell, H. N., Dugan, R. S., \& Stewart, J. Q. 1938, Astronomy II: Astrophysics \& Stellar Astronomy (Boston: Ginn \& Co.)

Sanduleak, N., \& Stephenson, C. B. 1973, ApJ, 185, 899

Scarfe, C. D., Batten, A. H., \& Fletcher, J. M. 1990, Publ. Dominion Astrophys. Obs. Victoria, 18, 21

Schmid, H. M. 1989, A\&A, 211, L31

Schmid, H. M., \& Schild, H. 2002, A\&A, 395, 117

Schmutz, W., Schild, H., Mürset, U., \& Schmid, H. M. 1994, A\&A, 288, 819

Seaquist, E. R., Krogulec, M., \& Taylor, A. R. 1993, ApJ, 410, 260

Skopal, A., Vanko, M., Pribulla, T., Chochol, D., Semkov, E., Wolf, M., \& Jones, A. 2007, Astron. Nachr., 328, 909

Skrutskie, M. F., et al. 2006, AJ, 131, 1163

The, P.-S. 1966, Contrib. Bosscha Obs., 34, 1

van Belle, G. T., et al. 1999, AJ, 117, 521

Vassiliadis, E., \& Wood, P. R. 1993, ApJ, 413, 641

Verbunt, F., \& Phinney, E. S. 1995, A\&A, 296, 709

Wallerstein, G. 1981, Observatory, 101, 172

Webster, B. L., \& Allen, D. A. 1975, MNRAS, 171, 171

Wray, J. D. 1966, PhD thesis, Northwestern Univ.

Zahn, J.-P. 1977, A\&A, 57, 383

Zamanov, R. K., Bode, M. F., Melo, C. H. F., Bachev, R., Gomboc, A., Stateva, I. K., Porter, J. M., \& Pritchard, J. 2007, MNRAS, 380, 1053 